\title{
The impact of itch symptoms in psoriasis: results from physician interviews and patient focus groups Denise Globe*1, Martha S Bayliss $^{2}$ and David J Harrison ${ }^{1}$
}

Address: ${ }^{1}$ Amgen Inc, One Amgen Center Drive, Thousand Oaks, CA, 91320 USA and ${ }^{2}$ Mapi Values, 3rd Floor 133 Portland Street, Boston, MA 02114, USA

Email: Denise Globe* - dglobe@amgen.com; Martha S Bayliss - martha.bayliss@mapivalues.com; David J Harrison - davidh01@amgen.com

* Corresponding author

Published: 6 July 2009

Health and Quality of Life Outcomes 2009, 7:62 doi:10.1 I86/1477-7525-7-62

This article is available from: http://www.hqlo.com/content/7/l/62

This is an Open Access article distributed under the terms of the Creative Commons Attribution License (http://creativecommons.org/licenses/by/2.0), which permits unrestricted use, distribution, and reproduction in any medium, provided the original work is properly cited.

\begin{abstract}
Background: The objective of this qualitative study was to better understand the impact of psoriasis symptoms using a 3-part process: I) develop a disease model for psoriasis to identify the most important concepts relevant to psoriasis patients; 2) conduct interviews with dermatologists to identify key areas of clinical concern; and 3) explore psoriasis patients' perceptions of the impact of psoriasis.
\end{abstract}

Methods: A disease model was developed from a review of the published literature and later revised based on the findings of clinician interviews and patient focus groups. To confirm the clinical relevance of the concepts identified in the disease model, 5 dermatologists were selected and interviewed one-on-one. They were asked to rate major psoriasis symptoms according to importance and bothersomeness level to patients on separate scales of I to I0. Results of clinician interviews were used to develop interview guides for patient focus groups. To identify important domains of psoriasis, 39 patients participated in 5 separate concept elicitation focus groups. Four focus groups included patients with severe psoriasis $(n=3 I)$ and one included patients with mild psoriasis $(n=8)$. Patients were asked to describe their current psoriasis symptoms and to rate them on a scale of I to 10, according to importance, severity, and troublesomeness. An average mean rating was calculated for each symptom throughout all focus groups.

Results: Clinicians most frequently mentioned itch $(n=5)$, psoriatic arthritis or "joint pains" $(n=$ $4)$, flaking $(n=4)$, and pain $(n=3)$ as primary physical symptoms of psoriasis. Three clinicians gave a rating of 10 for the importance of itch; two clinicians gave ratings of 8 and 7 for importance. The majority of patients rated itch as the most important (3I/39), most severe (3I/39), and most troublesome (24/39) symptom and noted that itch negatively impacted daily activities (eg, concentration, sleep, ability to attend work or school), as well as emotions (eg, anxiety and embarrassment).

Conclusion: These analyses suggest that itch is one of the most important symptoms of psoriasis, contributing to diminished health-related quality of life (HRQoL) in patients with both mild and severe disease. 


\section{Background}

Psoriasis is a chronic, systemic, inflammatory disease characterized by erythematous, scaly plaques that can itch and bleed. It has a prevalence of approximately $2.2 \%$ in the U.S. [1]. Psoriasis has a major impact on healthrelated quality of life (HRQoL) that is comparable to other major medical diseases such as cancer, arthritis, hypertension, heart disease, diabetes, and depression $[2,3]$. Dysregulation of the immune system (eg, T cells and cytokines), intrinsic epidermal components (eg, keratinocyte growth and differentiation factors), and environmental factors (eg, stress, cold weather, excessive alcohol intake, and some medications) contribute to the chronic, relapsing nature of the disease [4-6].

Psoriasis is a highly symptomatic disease. Symptoms of psoriasis include burning, stinging, inflammation, redness, itching, pain, scaling, and cracking of skin. Some symptoms (itch, soreness, pain, stinging) are included in the Dermatology Life Quality Index (DLQI), a 10-item questionnaire commonly used in clinical trials to assess HRQoL [7]. Although the DLQI is a good indicator of overall HRQoL and has been validated for use in patients with psoriasis [8], it does not assess all symptoms and does not provide a measure of symptom severity. Additional dermatology-specific and psoriasis-specific instruments include the Dermatology Quality of Life Scales (DQOLS), the Dermatology Specific Quality of Life (DSQL), Skindex, the Psoriasis Disability Index (PDI), and the Psoriasis Life Stress Inventory (PLSI) [9]. Other instruments used to assess HRQoL in dermatology clinical studies, the Short Form 36 health survey (SF-36) and the EuroQOL 5D (EQ-5D), are not specific for dermatologic diseases and do not provide information on severity, importance, or improvement of specific symptoms [8].

The objective of this qualitative study was to better understand the impact of psoriasis symptoms using a 3-part process: 1) develop a disease model for psoriasis to identify the most important concepts relevant to psoriasis patients; 2) conduct interviews with dermatologists to identify key areas of clinical concern; and 3) explore psoriasis patients' perceptions of the impact of psoriasis. Fulfillment of these objectives will satisfy the first component of the U.S. Food and Drug Administration (FDA) draft guidance on patient-reported outcome (PRO) development [10].

\section{Methods}

\section{Disease model}

The intent behind the disease model was to depict a broad overview of the issues encompassing the entire spectrum of psoriasis and to diagrammatically illustrate the interrelationships between these issues. Risk factors, symptoms, and treatments associated with psoriasis provided the concepts for the disease model, which was also intended to characterize the impact of psoriasis on patient functioning and well-being.

We developed a preliminary disease model from a review of the published literature pertaining to symptoms, severity, and impact of psoriasis. The preliminary disease model, based on information gathered in the literature search, was then revised based on physician and patient feedback.

We conducted the literature search using Medline $e^{\circledR}$ and the Mapi Research Trust database (a collection of articles composed of more than 15,580 articles on health outcomes assessment focusing on HRQoL and PROs). The following keywords were used in the Medline ${ }^{\circledast}$ search strategy: "psoriasis" or "dermatology" or "psoriatic nail dystrophy" or "plaque psoriasis" or "flexural psoriasis" or "guttate psoriasis" or "pustular psoriasis" or "nail psoriasis" or "erythrodermic psoriasis" or "immune-mediated psoriasis" or "eczema" or "dermatology" (43,451 results); "itch" or "pain" or "irritation" or "discomfort" or "inflammation" or "distress" or "soreness" (494,017 results); "patient-reported" or "quality of life" or "health-related quality of life" or "patient-reported outcomes" (89,385 results); "physical impacts" or "social impacts" or "emotional impacts" or "sexual impacts"; "clinician-reported" or "clinician-reported outcomes"; "questionnaire" or "patient-reported" or "instrument" or "scale" or "measure" (97 results). The search was limited to English language articles published after 1990.

\section{Physician Interviews}

Five dermatologists with experience identifying, diagnosing, and treating patients with psoriasis were screened by Amgen Inc. for one-on-one interviews. All interviews were conducted over the phone by Mapi Values (Boston, MA) using an interview guide with open-ended and closedended questions.

Four of the clinicians had over 10 years of experience, and one clinician had 7 years of experience. Four of the clinicians practiced medicine in a research and teaching capacity, while the fifth clinician practiced in a teaching capacity. Two of the physicians worked in both inpatient and outpatient care settings, while the other 3 worked solely with an outpatient population. All participants stated that they were familiar with the following psoriasis treatment modalities: systemic therapies; phototherapy, laser-assisted phototherapy; biologic therapies; and all topical medications.

Each dermatologist was asked to describe his or her opinions and experience dealing with major psoriasis symptoms such as itch, pain, burning, and scaling. The clinicians rated the importance and level of bothersomeness of each symptom to their patients. They also evalu- 
ated the relevance of the symptoms to psoriasis, location on the body, severity, and treatment and management challenges.

All interviews were audio-recorded and transcribed verbatim. The Atlas.ti (Atlas.ti Scientific Software Development $\mathrm{GmbH}$, Berlin, Germany) qualitative analysis software program was utilized to analyze the clinician interview transcriptions. A codebook was developed and reviewed by all members of the research team. Sentence and paragraph segments of the transcribed verbatim interviews were then coded by a trained researcher and reviewed by a senior team member. Statistics to quantify inter-rater reliability were not calculated, but any discrepancies in coding were resolved through a discussion and consensusbuilding process. We performed a stratification analysis to identify patterns, themes, and key concepts, providing descriptive statistics (frequency and distribution of quotes, rank, or concordance correlations for assessing the relative distance or concordance between categories). This analysis identified the constructs and domains most important to the clinicians and most salient to the experience of psoriasis and its symptoms. We used the results of these interviews to develop the interview guides for subsequent patient focus groups.

\section{Patient Focus Groups}

We conducted concept elicitation focus group discussions to assess patients' experience with psoriasis in terms of symptoms of the condition and the impact on functioning and well-being. Participants for this study were identified through a commercial recruitment agency (Global Market Research Group, Murietta, CA), which enlisted general practitioners to help with patient recruitment following the approval of the study protocol by the Copernicus Group Independent Review Board (IRB). Recruiting clinicians provided patients with an information letter explaining study procedures, compensation, and right to withdraw from the study without penalty or change in medical care. We chose the general practitioner patient population because these patients were less likely to be receiving systemic therapy and more likely to be symptomatic, ensuring the participation of patients with the full breadth of severity of psoriasis.

Thirty-one patients with physician-confirmed severe psoriasis ( $\geq 10 \%$ body surface area [BSA] affected by psoriasis and Psoriasis Area and Severity Index [PASI] score $\geq 10$ ) participated in 4 focus groups ( 6 to 9 patients per group). Eight patients with physician-confirmed mild psoriasis $(\geq$ $3 \%$ BSA affected by psoriasis and PASI score $=3$ ) participated in an additional focus group. The aim of the focus groups was to collect participants' experiences with psoriasis; specifically, information about psoriasis symptoms and the impacts of those symptoms on their lives.
During the concept elicitation phase of the focus groups, patients were asked to report the psoriasis symptoms they were currently experiencing and describe how psoriasis impacted their daily activities. Participants were also probed about several well-known psoriasis symptoms if these symptoms were not already mentioned by patients spontaneously. Following the discussions, patients were then asked to rate their current psoriasis symptoms on a visual analogue scale of 1 to 10, based on importance, severity, and troublesomeness (symptoms that were not currently experienced were rated 0). All symptoms, both spontaneous and probed, were rated by focus group participants. The average mean rating was calculated for the importance of each symptom throughout all focus groups and combined into an overall average importance score for each symptom.

Audio- and video-recordings were collected from all focus groups and transcribed by a transcription agency (Fantastic Transcripts, Boston, MA). The transcriptions were analyzed using Atlas.ti software. A codebook for patient focus groups, similar to the codebook that was devised for clinician interviews, was developed by the research team. The transcriptions from the focus groups were coded by a trained researcher and reviewed by a senior member of the team. Discussion and consensus-building were used to resolve coding discrepancies. We used descriptive statistics to characterize demographics, socioeconomic status, and disease characteristics of the focus group participants.

We developed a saturation grid for patient feedback in order to summarize the concept elicitation information found during the qualitative analysis. Conceptual saturation refers to the endpoint in the qualitative data collection and analysis process when further data collection and analysis cease to generate any new or distinctive categories, high level concepts, or substantive codes [11]. As opposed to quantitative analysis, in which an ideal sample size is calculated before data collection based on previous research and literature review, final sample size in qualitative research is driven by the prospective discovery of concepts, and hence there is no specific number that represents an "ideal" sample size [12].

We grouped the responses from each focus group by domain, sub-domain, and patient. The number of elicited responses were categorized by concept and reviewed across patients for each focus group. To construct the grid, the results for each concept from one focus group were compared to the results from a second focus group, based on the constant comparative method [13]. The totals for each concept from the first two focus groups were then compared to the third focus group. Next, the totals for each concept from the first three focus groups were compared to the fourth focus group. Finally, the totals from 
the first four focus groups for each concept were compared to the fifth focus group totals. Saturation is reached when subsequent focus groups or interviews do not yield any new information or concepts. The sample size of 39 patients was deemed to be adequate to achieve saturation based on established guidelines [14].

\section{Results}

\section{Disease Model}

The disease model (Figure 1) depicts a broad, big-picture view of issues related to psoriasis and the hypothesized inter-relationships. The major components of the disease model include: risk factors for psoriasis, psoriasis diagnosis, signs, symptoms, external factors, treatment, patientreported HRQoL outcomes, clinician-reported outcomes, and physiological/biological outcomes. In this disease model, each box is linked, either directly or indirectly, to every other box. The arrows in the model illustrate direct linkage between different aspects of the disease and treatment. Because every box in the disease model is linked in varying degrees to every other box, it is impracticable to generate a schematic including all possible relationships among the different aspects of psoriasis; consequently this disease model includes the relationships of primary importance. Our analyses focused on the impact of patient-reported symptoms (center of the model) on the patient-reported HRQoL domains most proximal to the patients' experiences (right side of the model).

The disease model begins with a diagnosis of psoriasis. The signs and symptoms of the disease are determined upon presentation to the physician and subsequent diag-

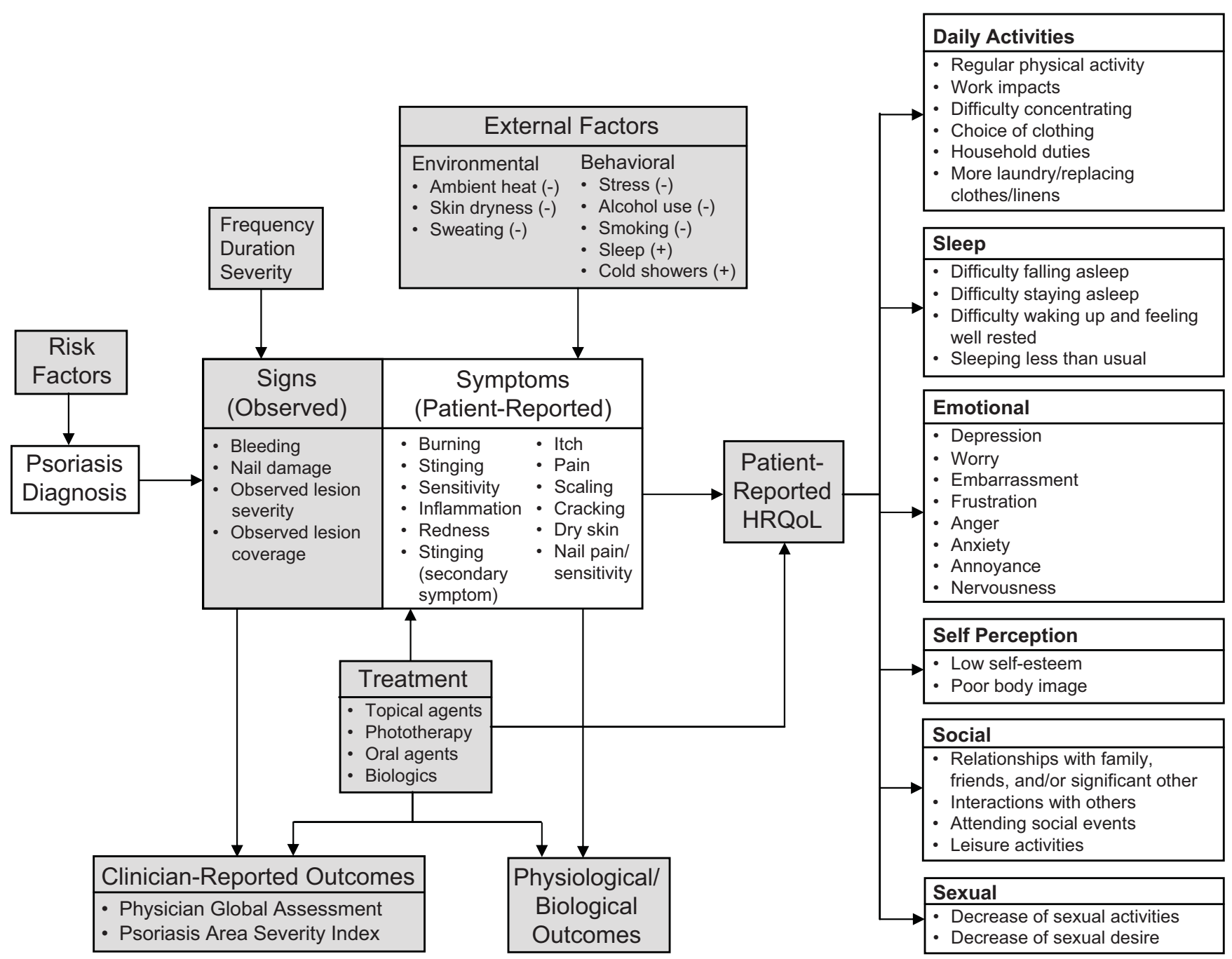

Figure I

Disease model of psoriasis. The model illustrates the relationship among symptoms of psoriasis and their impacts on patients' everyday lives. Shaded boxes represent aspects of psoriasis included in the disease model for comprehensiveness, but were not evaluated as a part of this study. 
nosis. How these signs and symptoms are interpreted by the patient and the clinician is dependent upon their frequency, duration, and severity. Important additions to the disease model based on patient feedback included symptoms (skin cracking and dry skin), daily activities (choice of clothing, work impacts, household duties, and more laundry/replacing clothes/linens), sleep impact (difficulty waking up and feeling well rested; sleeping less than usual), emotions (embarrassment, frustration, anger, anxiety, annoyance, and nervousness), and social impact (interaction with others, attending social events, leisure activities, and sexuality).

\section{Physician Interviews}

The most frequently mentioned symptoms reported by the clinicians were itch $(\mathrm{n}=5)$, psoriatic arthritis (or "joint pains"; $\mathrm{n}=4)$, soreness or pain $(\mathrm{n}=3)$, and flaking $(n=4)$. In terms of importance, the symptom of itch was rated a 10 by 3 clinicians and an 8 and a 7 by the 2 remaining clinicians, with 10 indicating the highest rank for importance. Three clinicians rated itch as the most bothersome symptom, though one of those clinicians rated itch, pain, burning, stinging, and flaking as equally bothersome symptoms to patients.

Exemplary quotes from clinician interviews regarding itch are presented in Table 1. Clinicians reported the location of itch was most commonly the scalp, anterior legs, the shins, groin, armpits, and also the back. Itch was found to be mostly associated with lesions on the body surface, though not exclusively. Clinicians also reported challenges to treating and managing itch symptoms, as there are no specific oral anti-itch medications and topical therapies lose effectiveness over time.

Table I: Select Comments Regarding Itch Symptoms from Clinician Interviews

Code Clinician Comments

Assessment of Severity

Itch

Relevance of itch

Location

Treatment/management challenges
"By how much the patient describes the itch. Like, if they say, it wakes me up at night, or it keeps me up at night, or it's relentless, or it drives me crazy, I know the itch is severe. Plus, you know, I might ask the patient to grade it on a scale of one to ten, how bad is your itch, with ten being the worst, one is being pretty mild or not present. (...) Oh, just the one to ten scale, that's the only systematic way I can grade itch numerically."

"Well, I don't have a scale typically in the clinic. There is no numeric scale that I use, but I go by whether it keeps them up at night, or not. That's typically the threshold. The severity of itching really keeps a patient aware at night, and that's about it. I know that's very subjective, but I don't use anything more quantifiable than itch."

"In psoriasis it's less than it is for atopic dermatitis. It doesn't usually keep them up at night, but it is, in some people, a symptom that is annoying, rather than disabling."

"If they're able to sleep at night, or it disables them during the daytime, if they've got to stop what they're doing to scratch. I also - well, I look at their skin to see if there are scratch marks, and if there's other accompanying signs of what we call excoriations, where they're digging at their skin, scratching and digging at their skin."

"Itch is a very bothersome symptom for psoriasis and other skin conditions such as eczema. And itch can be more problematic than pain. Itch is constant. Itch is something patients are much more aware of, and they're always scratching themselves to the point where they start to bleed, and their skin gets infected. And it's usually worse at nighttime, because patients are more aware about their body, as opposed to daytime, where people are more - you know, they're more busy at work and things. That's when then get home, then they start to itch more. But I would say itch by far is the number one factor that drives patients crazy."

"I think it's highly relevant, because it's probably the most day in, day out thing that a lot of people face in that the itching is - comes to the top of forefront of symptomology, whether it's itching in your scalp, or itching on the patches of psoriasis. And it's again probably one of the single most bothersome things, other than the fact that it's there."

"Scalp - wherever the plaques are, but scalp drives them crazy. Anywhere on the body."

"Is the itch from lesions? Usually, yes."

"Commonly hands, knees, ankles."

"Their scalp and their lower legs. It can be where there's no lesions in the scalp, but in the lower legs there usually some evidence of dry, scaling skin with some scratch marks."

"Like if the treatment works, that's great, but if it doesn't work, then we've got to keep adding things to help control the itch, whether it be a pill for itch that they take at nighttime, like an antihistamine, or using various creams to keep applying to control the itch... I would probably say maybe $60 \%$ of the time the itch can be controlled with one treatment, whether it be the shots or light therapy. And then $40 \%$ of the time it's not adequate, it's got to add in something else, like a topical cream, to control the itch." "Particular challenges (related to the treatment or management of the itch)? Again, I think it's related to treating the underlying psoriasis itself."

"Yes, there's no specific oral anti-itch medication. And some of the topical anti-itch medications are not always effective for long periods of time over large areas of the skin. Effective or feasible. If they have total body involvement, it's hard to - lotions, anti-itch lotions all over their body." 


\section{Patient Focus Groups}

A total of 39 patients participated in the focus groups (Table 2). The age and gender distribution of the patients who participated in the study were representative of the psoriasis population, although the group of patients with severe disease had a slightly lower percentage of women (55\%) than the group with mild disease (63\%). Also, the mean age was lower in the group with mild disease (36 years) than in the group with severe disease ( 45 years).

Exemplary quotes from the patient focus groups regarding the importance of itch in their everyday lives are presented in Table 3. When asked about the impact of psoriasis, patients with severe disease reported that itch symptoms affected concentration ( $n=3)$, and regular physical activity $(n=3)$. All patients with severe disease reported that itching or scratching their psoriasis impacted their sleep quality. Five patients reported missing days at work or school because of itch symptoms.

Patients with mild disease also reported difficulty concentrating due to itching or scratching, as well as knowing other people could see their psoriasis on their body. Two patients with mild disease mentioned that psoriasis affected them at work because of scratching. One patient spontaneously reported experiencing difficulty falling asleep because of itch symptoms. When probed, patients with mild disease reported that their difficulty sleeping was often due to scratching caused by their psoriasis.
The majority of patients rated itch as the most important and troublesome symptom (Table 4 ). The rating of itch as the most troublesome symptom was highest in patients with mild disease. The rating of itch as the most severe symptom was higher in patients with severe disease (mean rating 8.1 with $48 \%$ of patients rating the highest score of 10 ) than in patients with mild disease (mean rating 5.8 with $0 \%$ of patients rating the highest score of 10 ).

Concepts that were added to the disease model based on patient feedback and saturation grid analysis are shown in Table 5. Results of the saturation analysis showed that no new sub-concepts were mentioned by patients in the final focus group. Therefore, it was concluded that saturation was reached in the focus groups.

\section{Discussion}

The disease model of psoriasis developed here represents a global picture of the concepts and domains of psoriasis while providing support for patient-based assessments as endpoints in clinical trials. Itch was identified as a characteristic symptom of psoriasis in the literature review and included as one of the patient-reported symptoms of psoriasis in the disease model. The majority of physicians and patients indicated that itch was the most important symptom of psoriasis. Clinicians agreed in their interviews with the relevance of itch as an important symptom in psoriasis. Furthermore, physicians noted that itch can often be recalcitrant to traditional treatments. In assessing the

Table 2: Demography, Disease Diagnoses, and Health Status of Participants in Patient Focus Groups ${ }^{a}$

\begin{tabular}{lcc}
\hline & \multicolumn{1}{c}{ Focus Groups } \\
\cline { 2 - 3 } & Patients with Mild Psoriasis (N = 8) & Patients with Severe Psoriasis (N = 3 I) \\
\hline Female sex, $n$ (\%) & $5(63)$ & $17(55)$ \\
Age, mean years (range) & $36(20-74)$ & $45(22-66)$ \\
Race/Ethnicity, n (\%) & $5(63)$ & $24(77)$ \\
White & 0 & $4(13)$ \\
Black/African American & $1(13)$ & $1(3)$ \\
Hispanic & $1(13)$ & 0 \\
Mexican-American & $1(13)$ & 0 \\
Arabic & 0 & $1(3)$ \\
Spanish & & $(3)$ \\
Disease Diagnosis, $n$ (\%) & $(100)$ & $29(94)^{\mathrm{b}}$ \\
Psoriasis & 0 & $1(3)$ \\
Co-existing Psoriatic arthritis & $1(13)$ & $3(10)$ \\
Heath Status, $n$ (\%) & $4(50)$ & $9(29)$ \\
Excellent & $3(38)$ & $13(42)$ \\
Very good & 0 & $4(13)$ \\
Good & 0 & $2(7)$
\end{tabular}

aData not available for all participants for all characteristics so columns may not add up to $100 \%$.

bTwo patients did not fill out a page of the demographic form that included diagnosis of psoriasis. The clinicians for these patients confirmed the diagnosis of psoriasis.

$\mathrm{N}=$ total number of patients; $\mathrm{n}=$ number of patients for whom data are available 
Table 3: Select Comments Regarding Itch Symptoms from Patient Focus Groups

\begin{tabular}{|c|c|}
\hline Code/domain & Patient Comment (Severity of Psoriasis) \\
\hline Symptoms/itch & $\begin{array}{l}\text { "You itch a lot. Scratch a lot, I mean." (Severe) } \\
\text { "Scratch to relieve." (Severe) } \\
\text { "It will spread over my whole body, the itching. I'll get scabs on my knees, on my } \\
\text { elbows that (...) like yours (...) are very, very bad, and the more you scratch it, the } \\
\text { worse it gets. (...) it just spreads." (Severe) } \\
\text { "The worst is, for me, is just the itching. The itch and the dry." (Severe) } \\
\text { "My itching, it gets inflamed. (...) It spreads - (...) And the more you scratch - you're } \\
\text { scratching - (...) Mainly the itching." (Mild) } \\
\text { "Because I'm scratching everywhere, people not knowing the reason why I'm } \\
\text { scratching." (Mild) }\end{array}$ \\
\hline Change of psoriasis symptoms due to anxiety/worsen & $\begin{array}{l}\text { "So that's the worst, that's when I start to get really anxious. And it, like I said, it can } \\
\text { change over the course of a day. When I know I got to start getting ready for work, } \\
\text { that puts me in a whole other frame of mind and I will notice that I'm itching a little } \\
\text { bit more and or maybe my hands are not as calm as they are on my days off." } \\
\text { (Severe) }\end{array}$ \\
\hline Change of psoriasis symptoms due to stress/worsen & $\begin{array}{l}\text { "Mine only itches when I'm under stress." (Severe) } \\
\text { "...l'll be thinking about it way too much, and then I'll start getting - affecting my } \\
\text { skin, because the stress will make it outbreak, and then the outbreak, l'll want to } \\
\text { itch, and just scratch..." (Severe) }\end{array}$ \\
\hline Change of psoriasis symptoms over day/varies & $\begin{array}{l}\text { "Yeah, for me it changes a lot too... Towards the end of the night is when it's usually } \\
\text { more itchy for me." (Severe) }\end{array}$ \\
\hline Change of psoriasis symptoms over week/varies & $\begin{array}{l}\text { "...and then Sunday will hit, and then that's it. Then I'll start itching again, so it's like } \\
\text { - and Mondays are so busy at work. And like towards Wednesday - that's - I don't } \\
\text { know. It's like Monday and Wednesday. Those two days that - I don't know why } \\
\text { those - hate those days. (Severe) } \\
\text { "The itching to me varies. (...) Just sometimes I don't even think about it and other } \\
\text { times it just, boom, it's just itching." (Severe) }\end{array}$ \\
\hline Impact on daily activities/difficulty concentrating & $\begin{array}{l}\text { "You lose concentration, because you want to scratch and (...) really want to itch } \\
\text { this, but you don't want to itch it in front of somebody, and so you trail off to what } \\
\text { you were originally helping somebody with, if you're working." (Mild) } \\
\text { "Lack of concentration, or itchy - if you're really over-itchy, sometimes it's hard to } \\
\text { concentrate on something else other than that whole-body itch." (Severe) }\end{array}$ \\
\hline Impact on daily activities/choice of clothing & $\begin{array}{l}\text { "For some reason whenever I have anything that's } 100 \% \text { cotton I tend to itch more. } \\
\text { It gets irritated more, so everything is based on clothing or cotton. I try to buy a } \\
\text { certain type of clothes. So and that's what I got to wear all the time." (Severe) }\end{array}$ \\
\hline Impact on emotions/embarrassed & $\begin{array}{l}\text { "I'm in front of people all day long, and it's incredibly embarrassing to start bleeding } \\
\text { in front of someone, or scratching uncontrollably when you're not even thinking } \\
\text { about it." (Severe) } \\
\text { "Mine is just more of embarrassment. When you're scratching and people see } \\
\text { things coming off of you or on your clothing..." (Mild) }\end{array}$ \\
\hline Impact on sleep/difficulty falling asleep & $\begin{array}{l}\text { "Before you go to sleep yeah. (...) Because your itches." (Mild) } \\
\text { "It's itching instead of falling asleep." (Severe) } \\
\text { "It's falling asleep, when it's itching, and you - and then the minute you start } \\
\text { scratching, it only makes it worse. But it's hard...You tell yourself not to scratch, but } \\
\text { you think you're going to stop it, you know? And then you scratch it, it only makes } \\
\text { it worse, and you want to scratch more, and scratch more. It's bad." (Severe) }\end{array}$ \\
\hline Impact on sleep/difficulty staying asleep & $\begin{array}{l}\text { "Well, no. I'm going to wake up, I'm itchy. I'm going to put some cortisone on, I'm } \\
\text { going grease myself down - then I'm going to try and go back to bed." (Severe) } \\
\text { "Just, I want to rip my skin off, because it wakes me up. It's like it's never-ending." } \\
\text { (Severe) }\end{array}$ \\
\hline Miss days of school or work because of psoriasis/itch & $\begin{array}{l}\text { "Yeah, you go every week and you get shots to stop you from itching." (Severe) } \\
\text { "And I had it on my feet really bad, and I actually missed several days of work... } \\
\text { Well, it's the itching." (Severe) } \\
\text { "I can't just go out there (...) taking the train to work, being around a bunch of } \\
\text { people, and coming back home - the whole time I just want to scratch and itch..." } \\
\text { (Severe) }\end{array}$ \\
\hline
\end{tabular}

three qualities of itch (importance, severity, and troublesomeness), patients with severe psoriasis rated itch as more severe on the 10-point visual analogue severity scale and were more likely to select the most severe anchor of 10 (48\% with severe psoriasis compared with $0 \%$ of mild psoriasis).
Itch, or pruritus, has been shown to be one of the most embarrassing [15] and distressing [16] symptoms for patients with psoriasis. This diagnostic symptom of psoriasis has profound effects on HRQoL [2,17-22]. The affective dimension of itch (described by patients as "unbearable," "worrisome," bothersome," and "annoy- 
Table 4: Importance, Severity, and Troublesomeness of Itch Symptoms Rated by Patients With Mild and Severe Psoriasis

\begin{tabular}{|c|c|c|c|}
\hline & $\begin{array}{l}\text { Patients with Mild Disease } \\
\qquad(\mathbf{N}=\mathbf{8})\end{array}$ & $\begin{array}{l}\text { Patients with Severe Disease } \\
\qquad(\mathbf{N}=31)\end{array}$ & All Patients $(\mathbf{N}=39)$ \\
\hline \multicolumn{4}{|c|}{$\begin{array}{l}\text { Patients rating itch as the most important } \\
\text { symptom }^{\mathrm{a}}\end{array}$} \\
\hline No. of patients, $n$ & 8 & 23 & 31 \\
\hline Mean rating & 9.1 & 8.5 & 8.7 \\
\hline Patients rating a $10, \%$ & $88 \%$ & $70 \%$ & $74 \%$ \\
\hline \multicolumn{4}{|c|}{$\begin{array}{l}\text { Patients rating itch as the most severe } \\
\text { symptomb }\end{array}$} \\
\hline No. of patients, $n$ & 8 & 23 & 31 \\
\hline Mean rating & 5.8 & 8.1 & 7.5 \\
\hline Patients rating a $10, \%$ & $0 \%$ & $48 \%$ & $35 \%$ \\
\hline \multicolumn{4}{|c|}{$\begin{array}{l}\text { Patients rating itch as the most } \\
\text { troublesome symptom }\end{array}$} \\
\hline No. of patients, $n$ & 8 & 16 & 24 \\
\hline Mean rating & 9.0 & 8.0 & 8.3 \\
\hline Patients rating a $10, \%$ & $63 \%$ & $50 \%$ & $54 \%$ \\
\hline
\end{tabular}

aSymptoms were rated from I (least important) to 10 (most important)

bSymptoms were rated from I (least severe) to 10 (most severe)

cSymptoms were rated from I (least troublesome) to 10 (most troublesome)

Table 5: Representative Spontaneous Responses From the Concept Elicitation Saturation Grid From Patient Focus Groups

\begin{tabular}{|c|c|c|c|c|c|}
\hline & Total Responses & FG I vs FG 2 & FG I-2 vs FG 3 & FG I-3 vs FG 4 & FG I-4 vs FG 5 \\
\hline \multicolumn{6}{|l|}{ Symptoms } \\
\hline Itch & 19 & 4 vs 4 & 8 vs 3 & II vs 5 & 16 vs 3 \\
\hline Bleeding & 13 & 3 vs 5 & 8 vs 1 & 9 vs 3 & $\mid 2$ vs $\mid$ \\
\hline Cracking & 12 & 0 vs 5 & 5 vs 4 & 9 vs 3 & 12 vs 0 \\
\hline Scaling & 12 & 2 vs 4 & 6 vs 4 & 10 vs 1 & II vs I \\
\hline Dry skin & 6 & I vs I & 2 vs 1 & 3 vs 3 & 6 vs 0 \\
\hline \multicolumn{6}{|l|}{ Impact on daily activities } \\
\hline Choice of clothing & 17 & 2 vs 6 & 8 vs 2 & 10 vs 4 & 14 vs 3 \\
\hline Effects on work & 9 & 3 vs 2 & 5 vs 0 & 5 vs 2 & 7 vs 2 \\
\hline More laundry/replacing clothes and linens & 2 & 2 vs 0 & 2 vs 0 & 2 vs 0 & 2 vs 0 \\
\hline Household duties & I & 0 vs I & I vs 0 & I vs 0 & I vs 0 \\
\hline \multicolumn{6}{|l|}{ Impact on social life } \\
\hline Interaction with others & 15 & 3 vs 5 & 8 vs 3 & II vs 3 & |4 vs 1 \\
\hline Attending social events & 5 & 0 vs 2 & 2 vs 2 & 4 vs 0 & 4 vs I \\
\hline Leisure activities & 4 & 2 vs 1 & 3 vs 0 & 3 vs 1 & 4 vs 0 \\
\hline \multicolumn{6}{|l|}{ Impact on sleep } \\
\hline Sleeping less than usual & 2 & 2 vs 0 & 2 vs 0 & 2 vs 0 & 2 vs 0 \\
\hline Difficulty waking up and feeling well rested & 1 & 0 vs 1 & I vs 0 & I vs 0 & I vs 0 \\
\hline \multicolumn{6}{|l|}{ Impact on emotions } \\
\hline Embarrassed & 17 & 5 vs 0 & 5 vs 3 & 8 vs 4 & 12 vs 5 \\
\hline Annoyed & 7 & 2 vs 2 & 4 vs 2 & 6 vs 1 & 7 vs 0 \\
\hline Frustrated & 4 & 0 vs 3 & 3 vs 0 & 3 vs 0 & 3 vs 1 \\
\hline Anxious & 2 & 0 vs 0 & 0 vs 0 & 0 vs 1 & | vs I \\
\hline Nervous & 1 & 0 vs 0 & 0 vs 1 & I vs 0 & I vs 0 \\
\hline \multicolumn{6}{|l|}{ Impact on sex } \\
\hline Sexual activities & 5 & 2 vs 0 & 2 vs 1 & 3 vs 0 & 3 vs 2 \\
\hline Decreased sexual desire & 2 & 2 vs 0 & 2 vs 0 & 2 vs 0 & 2 vs 0 \\
\hline
\end{tabular}

$\mathrm{FG}=$ Focus group 
ing"), but not the sensory dimension of itch (described by patients as "burning," "stinging," and "crawling like ants"), is a significant predictor of depression, distress, and sleep impairment in patients with psoriasis [20]. To date, the importance and relevance of itch as a symptom in psoriasis patients has not been systematically assessed. Itch therefore represents an important PRO in clinical trials that should be considered when assessing the efficacy of treatment. Our study was not designed to determine causality between components (eg. symptoms and HRQoL) of the disease model. Better measures of itch should provide further insight into the relationships between triggers and aggravating factors and itch, as well as the effects of itch on HRQoL.

The results of the focus groups in this study are consistent with data from other focus groups [19] and a questionnaire-based study [22] that have documented the importance of itch to patients with psoriasis. Also consistent with these previously reported studies were the patients' identification of stress as an aggravating factor for itch and the negative impact of itch on sleep $[19,22]$. The results of these studies confirm the link between the impact of psoriasis symptoms and HRQoL in patients with psoriasis, as demonstrated in the disease model of psoriasis.

PROs used in clinical trials can provide fundamental information from the patients' perspective about the symptoms of psoriasis and the subsequent impacts that symptoms have on patients' lives. PROs are also fundamental in evaluating treatments in clinical trials to support approval, develop labeling, and substantiate potential advertising claims from a regulatory perspective. The results of our study demonstrate that itch matters to patients and clinicians, and assessment of itch should be included as a PRO in clinical trials of drugs used to treat psoriasis. The instrument used to assess itch should be clinically meaningful and be validated for reliability and responsiveness $[23,24]$. Other components of the disease model are also important; however, many of the items related to HRQoL can be captured in existing measures, such as the DLQI, SF-36, and EQ-5D.

The disease model of psoriasis developed in this study, in addition to the data from the physician interviews and patient focus groups, establishes a framework for the use of a PRO based on itch in clinical trials of drugs to treat psoriasis, in accordance with the first component of PRO development as required by the U.S. FDA $[10,24]$. Additional steps are to adjust the conceptual framework and draft the PRO instrument; confirm the measurement model and assess other measurement properties; modify the instrument; and collect, analyze, and interpret data. In accordance with FDA guidelines [24], the disease model was constructed using data from an extensive review of the literature. Clinician interviews confirmed essential ele- ments of the disease model. Patient focus groups were used to prioritize aspects of psoriasis that are most relevant to patients, including the effects of itch on patients' everyday lives.

A limitation of this study was the nonrandom convenience sampling of both physicians and patients, which may not be representative of all dermatologists or patients with psoriasis. Qualitative research by its very nature requires small sample sizes, limiting the generalizability of the findings. Additionally, this study focused on symptoms of psoriasis; other domains in the disease model are equally important in understanding the impact of psoriasis on patients' lives but were outside the scope of these analyses.

Despite the small sample size, the entry criteria for participation in the study were similar to those used in clinical trials, increasing the likelihood of generalizability of our findings to other patients with psoriasis. All of the patients had physician-confirmed disease and represented the spectrum of disease severity in clinical practice. Because saturation was reached in the grid analysis, the sample size was adequate to meet our objective of achieving a better understanding of the impact of psoriasis on patients' lives.

Our results suggest that improvements in itch will result in improvements in patients' HRQoL. An anti-psoriatic drug that improves the symptoms of itch could therefore support a claim that the drug also improves HRQoL. The disease model reinforces the importance of measuring the components of disease as well as the patients' perspective within the clinical trial setting. In addition to the visual analog scale of itch severity frequently used in clinical trials, our study supports the development of itch questionnaires, such as the one developed by Yosipovitch et al [25], to fully assess the impact of itch on HRQoL in patients with psoriasis.

\section{Conclusion}

These analyses enhanced our understanding of the impact of psoriasis symptoms on patients' lives, and suggest that itch is one of the most important symptoms of psoriasis, contributing to diminished HRQoL in patients with both mild and severe disease. Our results indicate a need for assessments of itch as well as skin lesions in clinical practice, and that itch should be considered as an endpoint in studies assessing the impact of disease and/or treatment in patients with psoriasis.

\section{Abbreviations}

BSA: Body surface area; HRQoL: Health-related quality of life; IRB: Independent review board; PASI: Psoriasis Area and Severity Index; PRO: Patient-reported outcome. 


\section{Competing interests}

DG and DJH are compensated employees and shareholders in Amgen Inc. MSB has received research funding from Amgen Inc.

\section{Authors' contributions}

DG made substantial contributions to the conception and design of the study and the interpretation of the data. MSB made substantial contributions to the conception and design of the study; and acquisition, analysis and interpretation of the data. DJH made substantial contributions to the interpretation of the data. All authors were involved in drafting the manuscript and revising it critically for important intellectual content, and have given final approval of the version to be published.

\section{Acknowledgements}

Funding for the study and manuscript preparation was provided by Amgen Inc. The authors thank Edward Mancini, DPM, of Amgen Inc. and Julia R.

$\mathrm{Gage}, \mathrm{PhD}$, on behalf of Amgen Inc. for assistance with the preparation of the manuscript.

\section{References}

I. Stern RS, Nijsten T, Feldman SR, Margolis DJ, Rolstad T: Psoriasis is common, carries a substantial burden even when not extensive, and is associated with widespread treatment dissatisfaction. J Investig Dermatol Symp Proc 2004, 9(2): I36-139.

2. de Korte J, Sprangers MA, Mombers FM, Bos JD: Quality of life in patients with psoriasis: a systematic literature review. J Investig Dermatol Symp Proc 2004, 9(2): I40-I47.

3. Rapp SR, Feldman SR, Exum ML, Fleischer AB Jr, Reboussin DM: Psoriasis causes as much disability as other major medical diseases. J Am Acad Dermatol 1999, 4 I (3 Pt I):40 I-407.

4. Gaspari AA: Innate and adaptive immunity and the pathophysiology of psoriasis. J Am Acad Dermatol 2006, 54(3 Suppl 2):S67-80.

5. Tschachler E: Psoriasis: the epidermal component. Clin Dermatol 2007, 25(6):589-595.

6. Dika E, Bardazzi F, Balestri R, Maibach HI: Environmental factors and psoriasis. Curr Probl Dermatol 2007, 35: | |8-|35.

7. Finlay AY, Khan GK: Dermatology Life Quality Index (DLQI) a simple practical measure for routine clinical use. Clin Exp Dermatol 1994, 19(3):210-216.

8. Shikiar R, Willian MK, Okun MM, Thompson CS, Revicki DA: The validity and responsiveness of three quality of life measures in the assessment of psoriasis patients: results of a phase II study. Health Qual Life Outcomes 2006, 4:7I.

9. Finlay AY: Quality of life assessments in dermatology. Semin Cutan Med Surg 1998, 17(4):291-296.

10. Patrick DL, Burke LB, Powers JH, Scott JA, Rock EP, Dawisha S, O'Neill R, Kennedy DL: Patient-reported outcomes to support medical product labeling claims: FDA perspective. Value Health 2007, I0(Suppl 2):S125-137.

II. Mays N, Pope C: Rigour and qualitative research. BMJ 1995, 3II(6997): 109-II2.

12. Rowan M, Huston P: Qualitative research articles: information for authors and peer reviewers. CMAJ 1997, I 57( I 0): 1442- I 446.

13. Bowen GA: Naturalistic inquiry and the saturation concept: a research note. Qualitative Research 2008, 8(I): I37-I52.

14. Guest $G$, Bunce $A$, Johnson L: How many interviews are enough? An experiment with data saturation and variability. Field Methods 2006, I 8(I):59-82.

15. Kerkhof PC van de, de Hoop D, de Korte J, Cobelens SA, Kuipers MV: Patient compliance and disease management in the treatment of psoriasis in the Netherlands. Dermatology 2000, 200(4):292-298.
16. Kerkhof PC van de, de Hoop D, de Korte J, Kuipers MV: Scalp psoriasis, clinical presentations and therapeutic management. Dermatology 1998, 197(4):326-334.

17. Dawn A, Yosipovitch G: Treating itch in psoriasis. Dermatol Nurs 2006, I 8(3):227-233.

18. Verhoeven L, Kraaimaat F, Duller P, Kerkhof P van de, Evers A: Cognitive, behavioral, and physiological reactivity to chronic itching: analogies to chronic pain. Int J Behav Med 2006, 13(3):237-243.

19. Amatya B, Nordlind K: Focus groups in Swedish psoriatic patients with pruritus. J Dermatol 2008, 35(I): I-5.

20. Zachariae R, Zachariae CO, Lei U, Pedersen AF: Affective and sensory dimensions of pruritus severity: associations with psychological symptoms and quality of life in psoriasis patients. Acta Derm Venereol 2008, 88(2): | 21 - 127.

21. Hrehorów E, Reich A, Szepietowski J: Quality of life of patients with psoriasis: relationship with pruritus, stress and symptoms of depression. Dermatologia Kliniczna 2007, 9(I):19-23.

22. Amatya B, Wennersten G, Nordlind K: Patients' perspective of pruritus in chronic plaque psoriasis: a questionnaire-based study. J Eur Acad Dermatol Venereol 2008, 22(7):822-826.

23. Revicki DA, Erickson PA, Sloan JA, Dueck A, Guess H, Santanello NC: Interpreting and reporting results based on patient-reported outcomes. Value Health 2007, IO(SuppI 2):SI I6-I 24.

24. Burke LB, Kennedy DL, Miskala PH, Papadopoulos EJ, Trentacosti AM: The use of patient-reported outcome measures in the evaluation of medical products for regulatory approval. Clin Pharmacol Ther 2008, 84(2):28I-283.

25. Yosipovitch G, Goon A, Wee J, Chan YH, Goh CL: The prevalence and clinical characteristics of pruritus among patients with extensive psoriasis. $\mathrm{Br} /$ Dermatol 2000, I 43(5):969-973.
Publish with Bio Med Central and every scientist can read your work free of charge

"BioMed Central will be the most significant development for disseminating the results of biomedical research in our lifetime. "

Sir Paul Nurse, Cancer Research UK

Your research papers will be:

- available free of charge to the entire biomedical community

- peer reviewed and published immediately upon acceptance

- cited in PubMed and archived on PubMed Central

- yours - you keep the copyright
BiolMedcentral 